Scattering from two-phase media with singularities

Max Teubner

Citation: 91, (1989); doi: 10.1063/1.456993

View online: http://dx.doi.org/10.1063/1.456993

View Table of Contents: http://aip.scitation.org/toc/jcp/91/4

Published by the American Institute of Physics 


\title{
Scattering from two-phase media with singularities
}

\author{
Max Teubner \\ Max-Planck-Institute für biophysikalische Chemie, Postfach 2841, D-3400 Göttingen, Federal Republic of \\ Germany
}

(Received 1 March 1989; accepted 1 May 1989)

Some years ago Ciccariello et al.$^{1,2}$ have studied the effect of edges and contact points on the bulk scattering from two-phase media. Now there is a variety of systems, where the scattering length densities of the two media are equal, and only the interface scatters. Examples are foams or acqueous solutions of membrane-forming substances like surfactants and lipids. Another example is given by neutron scattering of microemulsions. If the scattering length densities of water and oil are matched by partial deuteration, only the surfactant film is observed. ${ }^{3}$ Since many of the systems are expected to display singularities of the above mentioned type, it seems useful to supply the corresponding results for surface scattering.

We start with the definition of the surface correlation function. ${ }^{4}$ Let $\delta_{s}^{\epsilon}(r)$ be the characteristic function for a thin sheet $S^{\epsilon}$ of uniform thickness $\epsilon$ approximating the surface $S$. For small $\epsilon, \epsilon^{-1} \delta_{S}^{\epsilon}$ is an approximation to $\delta_{S}$, the $\delta$ function concentrated on $S$. Then the surface correlation function is defined by

$$
g_{s s}\left(\mathbf{r}, \mathbf{r}^{\prime}\right)=\lim _{\epsilon \nmid 0} \epsilon^{-2}\left\langle\delta_{S}^{\epsilon}(\mathbf{r}) \delta_{S}^{\epsilon}\left(\mathbf{r}^{\prime}\right)\right\rangle .
$$

It can be proved ${ }^{4}$ that $g_{s s}$ is given by the following expression:

$$
g_{s s}\left(\left|\mathbf{r}, \mathbf{r}^{\prime}\right|\right)=\frac{1}{4 \pi\left|\mathbf{r}-\mathbf{r}^{\prime}\right|^{2}} \frac{1}{V} \int_{S} d S \int_{C_{r, r}} \frac{d s^{\prime}}{\left|\hat{\mathbf{r}} \times \mathbf{n}^{\prime}\right|}
$$

Here $\mathbf{r}$ ranges over $S, C_{r, r}$ is the curve common to $S$ and the sphere with center at $\mathbf{r}$ and radius $\left|\mathbf{r}-\mathbf{r}^{\prime}\right|, \hat{\mathbf{r}}=\left(\mathbf{r}-\mathbf{r}^{\prime}\right) /$ $\left|\mathbf{r}-\mathbf{r}^{\prime}\right|, \mathbf{n}^{\prime}$ is the normal on $S$ at $\mathbf{r}^{\prime}$, and $s^{\prime}$ is the arclength of $C_{r, r}$. For small $r$ one finds

$$
g_{s s}(r)=\frac{S}{V} \frac{1}{2 r} \text {. }
$$

For a smooth surface the next term is of order $r$ and has been calculated in Ref. 4. Edges or contact points generate additional terms of order $O(1)$. The calculation of these terms is similar to, but simpler than the corresponding calculation in Refs. 1 and 2 . Therefore we only present the results. If no contact points are present

$$
\lim _{r, 0}\left[g_{s s}(r)-\frac{S}{V} \frac{1}{2 r}\right]=\frac{1}{\pi V} \int_{L}\left(\frac{\alpha}{\sin \alpha}-1\right) d l,
$$

where the line integral is performed over all the edges in $V$ and $\pi \pm \alpha(-\pi<\alpha<\pi)$ represents the angle between the corresponding half-planes. (For $\alpha= \pm \pi$ the two halfplanes coincide. For $\alpha=0$ the surface is smooth.) For example, for a circular cylinder of radius $R$ and height $h$ one finds by direct evaluation for $r<\operatorname{Min}(2 R, h)$,

$$
\begin{aligned}
V g_{s s}(r)= & \frac{2 R^{2}}{r}\left[\arccos \frac{r}{2 R}-\frac{r}{2 R} \sqrt{1-\frac{r^{2}}{4 R^{2}}}\right]+\pi R \\
& -R \int_{0}^{\pi} \arcsin \left(\frac{r}{2 R} \sin \theta\right) \sin \theta d \theta \\
& +\frac{R}{r} \int_{0}^{\pi} \frac{h-r|\cos \theta|}{\sqrt{1-\left(r^{2} / 4 R^{2}\right) \sin \theta}} d \theta
\end{aligned}
$$

and for small $r$,

$$
V g_{s s}(r)=S \frac{1}{2 r}+4 R\left(\frac{\pi}{2}-1\right)+O(r)
$$

which coincides with Eq. (4).

If $N_{c}$ contact points per volume are present,

$$
2 \pi \frac{N_{c}}{V}\left\langle\frac{1}{\sqrt{ } \Delta}\right\rangle
$$

has to be added to Eq. (4). The average is performed over the the contact points and $\Delta$ is the Hessian of the difference surface at the contact point as defined in Ref. 2. For two spheres in contact

$$
\Delta^{-1 / 2}=\frac{R_{1} R_{2}}{R_{1}+R_{2}} .
$$

Transforming these results into $q$ space we find for the Fourier-transform $\chi_{s s}$ of $g_{s s}$,

$$
\begin{aligned}
\frac{1}{2 \pi^{2}} \int_{0}^{\infty}\left[q^{2} \chi_{s s}(q)-2 \pi \frac{S}{V}\right] d q \\
\quad=\frac{1}{\pi V} \int_{L}\left(\frac{\alpha}{\sin \alpha}-1\right) d l+2 \pi \frac{N_{c}}{V}\left(\frac{1}{\sqrt{ } \Delta}\right) .
\end{aligned}
$$

Note that the right-hand side is always nonnegative. For comparison I also quote the corresponding result ${ }^{1,2}$ for the bulk correlation function

$$
\begin{aligned}
& \frac{1}{2 \pi^{2}} \int_{0}^{\infty}\left[q^{4} \chi_{b b}(q)-2 \pi \frac{S}{V}\right] d q \\
& \quad=-\frac{1}{\pi V} \int_{L}(1-\alpha \cot \alpha) d l-4 \pi \frac{N_{c}}{V}\left\langle\frac{1}{\sqrt{ } \Delta}\right\rangle .
\end{aligned}
$$

If both surface and bulk scattering can be measured by contrast matching, it can be tested whether there are sharp edges in the system. If there are edges, and any one of the three quantities, $L, \alpha,\left(N_{c} / V\right)\langle 1 / V \Delta\rangle$ is known, the other two can be determined. For hard spheres, for example, only the contact points contribute to Eqs. (9) and (10). For an attractive interaction the concentration of contact points is exponentially dependent on the interaction potential at contact. In this way the contact potential can be measured by scattering techniques.

We finally mention a type of line singularities that may not be uncommon in surfactant solutions. If there is attrac- 
tion among the membranes, they will occasionally stick together in close contact over some extended regions. At the boundary they will in general depart tangentially forming a line of cusp singularities. If, for example, the membranes form flexible spheres as in vesicles or some microemulsions, an attraction may lead to dimers ${ }^{5}$ having a finite contact area. Such lines of cusp singularities produce in bulk scattering a term $\sim q^{-(4+1 / 2)}$ and in surface scattering a term $\sim q^{-(2+1 / 2)}$ in addition to the Porod terms $\sim q^{-4}$ and $q^{-2}$. The coefficients are $-2 \pi^{3 / 2}(L / V)\left\langle\left|k_{1}-k_{2}\right|^{-1 / 2}\right\rangle$ and $2 \pi^{3 / 2}(L / V\rangle\left\langle\left|k_{1}-k_{2}\right|^{-1 / 2}\right\rangle$, respectively, where $L$ is the length of the singularity line $L,\langle\rangle$ is the average along $L$, and $k_{1}, k_{2}$ are the sectional curvatures normal to $L$ of the two surfaces which join tangentially at $L$.

'S. Ciccariello, G. Cocco, A. Benedetti, and S. Enzo, Phys. Rev. B 23, 6474 (1981).

${ }^{2}$ S. Ciccariello and A. Benedetti, Phys. Rev. B 26, 6384 (1982).

${ }^{3}$ L. Auvray, J. P. Cotton, R. Ober, and C. Taupin, J. Phys. Chem. 88, 4586 (1984).

${ }^{4} \mathrm{M}$. Teubner, J. Chem. Phys. (to be published).

${ }^{5}$ L. A. Turkevich, in Physics of Complex and Supermolecular Fluids, edited by S. A. Safran and N. A. Clark (Wiley, New York, 1987), p. 241.

\title{
$\mathbf{N H}_{2}$ electron affinity
}

\author{
C. Tom Wickham-Jones, Kent M. Ervin, G. Barney Ellison, and W. Carl Lineberger \\ Department of Chemistry and Biochemistry, University of Colorado, and Joint Institute for Laboratory \\ Astrophysics, University of Colorado and National Institute of Standards and Technology, Boulder, \\ Colorado 80309
}

(Received 22 March 1989; accepted 20 April 1989)

We report the electron affinity of amidogen $\left(\mathrm{NH}_{2}\right)$, the amino radical, obtained from the photoelectron spectrum of the amide ion $\left(\mathrm{NH}_{2}^{-}\right)$. The literature electron affinity values from early laser photodetachment experiments have relatively large uncertainties: $\mathrm{EA}\left(\mathrm{NH}_{2}\right)=0.744 \pm 0.022 \mathrm{eV}$ from photodetachment threshold measurements ${ }^{1}$ and $\mathrm{EA}\left(\mathrm{NH}_{2}\right)=0.779 \pm 0.037 \mathrm{eV}$ from photoelectron spectroscopy. ${ }^{2}$ The development of higher resolution (5-25 $\mathrm{meV}$ ) negative ion photoelectron spectrometers ${ }^{3-6}$ now permits a significantly improved determination of the electron affinity as well as the observation of more detailed structure in the photoelectron spectrum. The spectrum reported here shows clear rotational bands in the photodetachment of $\mathrm{NH}_{2}^{-}$. Partially resolved rotational band structure has been observed previously in photoelectron spectra ${ }^{5,7}$ of small hydride ions such as $\mathrm{OH}^{-}, \mathrm{OD}^{-}, \mathrm{SH}^{-}, \mathrm{SD}^{-}$, and $\mathrm{CH}_{2}^{-}$.

The photoelectron spectrometer used in this experiment has been described in detail previously. ${ }^{5} \mathrm{NH}_{2}^{-}$is produced by introducing ammonia into a helium flow downstream of a microwave discharge; a beam of about $10 \mathrm{pA}$ was achieved. Negative ions are extracted from the flow tube, mass selected with a Wien velocity filter, and crossed with a continuous $363.8 \mathrm{~nm}(3.408 \mathrm{eV})$ laser beam of fixed photon energy at a power of $40 \mathrm{~W}$ in an optical build-up cavity. ${ }^{8}$ The kinetic energy of photodetached electrons is measured by a hemispherical electrostatic analyzer. ${ }^{5}$ The absolute electron kinetic energy (eKE) is calibrated with $\mathrm{O}^{-}$ions, $\mathrm{EA}(\mathrm{O})=1.461125 \pm 0.000001 \mathrm{eV},{ }^{9}$ and the energy scale compression factor ${ }^{5}$ of $0.6 \%$ is determined from photodetachment of $\mathbf{W}^{-}$. The uncertainty of the eKE scale is \pm 5 $\mathrm{meV}$ and the instrumental linewidth is $8-9 \mathrm{meV}$. The rota- tional temperature of $\mathrm{OH}^{-}$ions produced in this source under similar conditions is $315 \pm 20 \mathrm{~K}$, and the vibrational temperatures are typically $300-500 \mathrm{~K} .^{8}$

Figure 1 shows the $\mathrm{NH}_{2}\left(\widetilde{X}^{2} B_{1}, v^{\prime}=0\right) \leftarrow \mathrm{NH}_{2}^{-}\left(\widetilde{X}^{1} A_{1}\right.$, $v^{\prime \prime}=0$ ) vibrational origin transition in the photoelectron spectrum of $\mathrm{NH}_{2}^{-}$. We also observed transitions in the symmetric stretch $\mathrm{NH}_{2}\left(v_{1}^{\prime}=1\right) \leftarrow \mathrm{NH}_{2}^{-}\left(v_{1}^{\prime \prime}=0\right)$ and in the bend $\mathrm{NH}_{2}\left(v_{2}^{\prime}=1\right) \leftarrow \mathrm{NH}_{2}^{-}\left(v_{2}^{\prime \prime}=0\right)$ which had intensities of $0.5 \%$ and $0.9 \%$ respectively relative to the vibrational origin. Weak transitions to the $\widetilde{A}^{2} A_{1}$ state ${ }^{10}$ of $\mathrm{NH}_{2}$ were observed at electron kinetic energies below $1.5 \mathrm{eV}$. The rotational contour of the origin transition shows $Q, P$, and $R$ branches. The $Q$ branch has a FWHM of $8.5 \mathrm{meV}$, limited by the instrumental resolution. The rotationless origin is found from the rotational modeling to be within $1 \mathrm{meV}$ of the center of the $Q$ branch and this yields the electron affinity $\mathrm{EA}\left(\mathrm{NH}_{2}\right)=0.771 \pm 0.005 \mathrm{eV}$. This value for $\mathrm{EA}\left(\mathrm{NH}_{2}\right)$ falls within the range of the previous experimental determinations. ${ }^{1,2}$ A recent theoretical value ${ }^{11}$ is $\mathrm{EA}\left(\mathrm{NH}_{2}\right)=0.707$ $\mathrm{eV}$. We also measured the angular distribution of the ejected electron with the laser operating on the $351 \mathrm{~nm}$ line. This showed anisotropic detachment with asymmetry parameter $\beta$ of $0.34 \pm 0.05$. Within experimental error the $Q, P$, and $R$ branches all had the same angular distribution.

The ground electronic state of the amino radical is formed by removing an electron from the nonbonding $b_{1}$ orbital of $\mathrm{NH}_{2}^{-}$. The resulting small geometry change upon photodetachment is reflected by the narrowness of the $Q$ branch and by the weak intensities of higher vibrational transitions. The observed vibrational transitions were totally consistent with the known experimental molecular geome- 